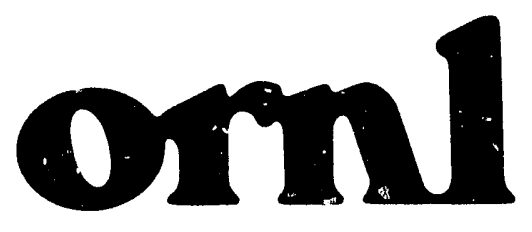

OAK RIDGE NATIONAL LABORATORY

MARTH MARIETTA

\title{
Discrete Pearson Distributions
}

K. O. Bowman

L. R. Shenton

M. A. Kastenbaum 
This report has reproduced directly from the best available copy.

Available to DOE and DOE contractors from the Office of Scientific and Technical Information, P.O. Box 62, Oak Ridge. TN 37831 ; prices available from (615) 576-8401, FTS 626-8401.

Available to the public from the National Technical Information Service, U.S. Department of Commerce, 5285 Port Royal Rd., Springtield, VA 22161.

This report was prepared as an account of work sponsored by an agency of the United States Government. Neither the United States Government nor any agency thereot, nor any of their employes, makes any warranty, express or implied, or assumes any legal liability or responsibility for the accuracy, completeness, or usefulness of any information, apparatus, product, or process disclosed, or represents that its use would not intringe privately owned rights. Reference herein to any specific commercial product, process, or service by trade name, trademiark. mariulacturer, or otherwise does not necessarily consti. tute or imply its endorsement, recommendation, or favoring by the United Siates Government or any agency thereof. The views and opinions of zuthors expressed herein do not necessarily state or reflect those of the United States Government or any agency thereot. 


\section{O]}

ORNL/TM- -11899

Engineering Physics and Mathematics Division

DE92 004700

Mathematical Sciences Section

\section{DISCRETE PEARSON DISTRIBUTIONS}

K.O. Bowman ${ }^{\dagger}$

L.R. Shenton :

M.A. Kastenbaum *

+ Mathematical Sciences Section

Oak Ridge National Laboratory

Oak Ridge, TN 37831-6367

\$ University of Georgia

Athens, GA 30602

* P.O. Box 315

Basye, VA 22810

Date Published: November, 1991

Prepared by the

Oak Ridge National Lauoratory

Oak Ridge, Tennessee 37831

managed by

Martin Marietta Energy Systems, Inc.

for the

U.S. DEPARTMENT OF ENERGY

under Contract No. DE-AC05-840R21400 
Contents

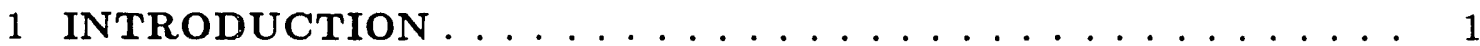

2 BASIC FORMULAE FOR THE SEMI-INFINITE CASE . . . . . . . 1

2.1 Development of Formulae . . . . . . . . . . . . 1

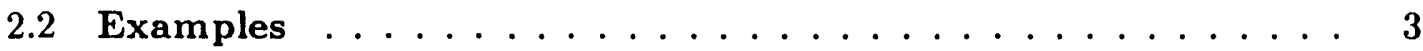

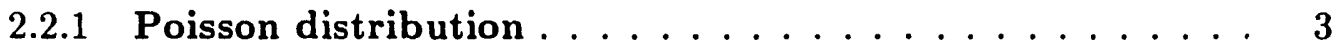

2.2.2 Stuttering Poisson Distribution ........... 3

2.2.3 Binomial distribution . . . . . . . . . . . . . 4

2.2.4 Ord's Example of Type I Distribution . . . . . . . . . . . 4

3 BASIC FORMULAE FOR THE BOUNDED CASE . . . . . . . . 5

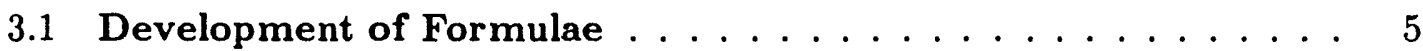

3.2 Examples .................... 7

3.2.1 Triangular Distribution ............. 7

3.2.2 Non-Symmetric Distribution . . . . . . . . . . 8

3.2.3 Truncated Poisson Distribution . . . . . . . . . . 9

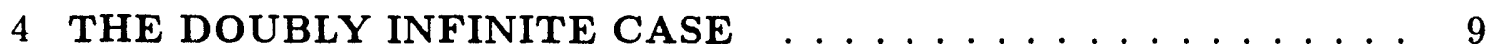

4.1 Formulae $\ldots \ldots \ldots \ldots \ldots$

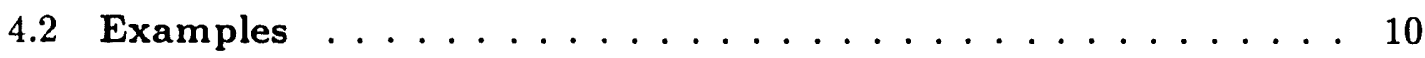

4.2 .1 Pearson Type IV Moments . . . . . . . . . . . . . 10

4.2.2 Discrete Pseudo-Normal Distribution . . . . . . . . . 10

4.2.3 Pearson Type VII Moments . . . . . . . . . . . 11

4.2 .4 Bessel Distribution . . . . . . . . . . . . . . . 11

4.2.5 Bessel Distribution with Bias . . . . . . . . . . 12

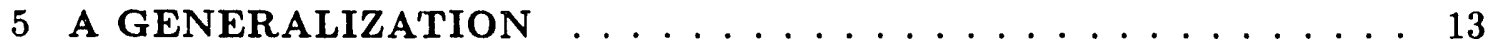

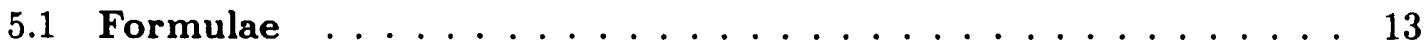

5.2 The Hansmann Distribution ............... 13

5.3 Further Comments on the Models . . . . . . . . . . . . . . 14

5.4 Examples . . . . . . . . . . . . . . . . 15

5.4.1 Discrete Pseudo-Normal Distribution . . . . . . . 15

5.4.2 Discrete Pseudo-Normal with the First 8 Moments . . . 15

5.4.3 Discrete Pseudo-Normal with the First 10 Moments . . . 16

5.4.4 Discrete Pseudo-Normal with the First 16 Moments . . . 17

5.4.5 Discrete Bessel Distribution with the First 10 Moments 17

6 THE ALGEBRAIC STRUCTURE FOR THE DOUBLY BOUNDED

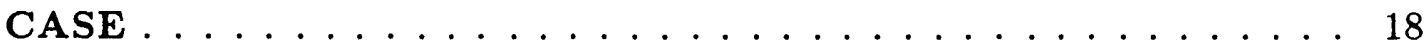

6.1 First Order Equations . . . . . . . . . . . . . . 18

6.2 Second Order Equations . . . . . . . . . . . . . . . . 19

7 THE EXPONEIJTIAL AND THE NORMAL . . . . . . . . . . 20

7.1 The Continued Fraction . . . . . . . . . . . . . . 20

7.2 Relation to Whittaker Functions .............. 21

7.3 Application to the Normal ............... 22 
7.4 Moments of the Discrete Normal Distribution . . . . . . . . . . 22

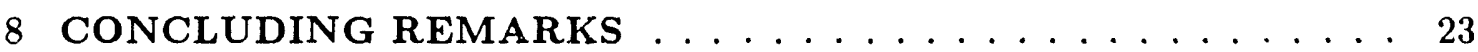

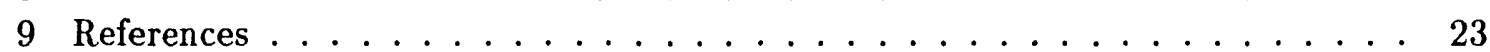




\section{Acknowledgements}

A research supported under contract ERD-89-828 with the Center for Indoor Air Research by Martin Marietta Energy Systems, Inc., under contract DE-AC05-840R21400 with the U.S. Department of Energy. 


\title{
DISCRETE PEARSON DISTRIBUTIONS
}

\author{
K.O. Bowman \\ L.R. Shenton \\ M.A. Kastenbaum
}

\begin{abstract}
These distributions are generated by a first order recursive scheme which equates the ratio of successive probabilities to the ratio of two corresponding quadratics. The use of a linearized form of this model will produce equations in the unknowns matched by an appropriate set of moments (assumed to exist). Given the moments we may find valid solutions. There are two cases; (a) distributions defined on the non-negative integers (finite or infinite) and (b) distributions defined on negative integers as well. For (a), given the first four moments, it is possible to set this up as equations of finite or infinite degree in the probability of a zero occurrence, the $s$ th component being a product of $s$ ratios of linear forms in this probability in general. For (b) the equation for the zero probability is purely linear but may involve slowly converging series; here a particular case is the discrete normal. Regions of validity are being studied.
\end{abstract}




\section{IIJTRODUCTION}

Being confrontec with several sets of extensive data of a discrete nature organized into cells, we found that none of the classical structures (Poisson, Binomial, Negative Binomial) came near to acceptability from a goodness-of-fit point of view. A search seemed to suggest that even though there are several generalizations, the fitting problem using moments implied fairly serious complications. Since we had 20 or more cells, it appeared that 4 or more parametered discrete distributions would be needed. It was natural to consider the Pearson discrete family generated by the 1st order recurrence

$$
y_{\tau}=\left\{\frac{P_{s}(r)}{Q_{t}(r)}\right\} y_{r-1}, \quad(r=1,2, \cdots)
$$

$P_{s}$ and $Q_{t}$ being polynomials in $r$, and the probabilities being $y_{0}, y_{1}, y_{2}, \cdots$ of occurrences in corresponding cells. If we are confronted with experimental (or even mathematically defined) data, then we can think of utilizing a set of moments (data moments will certainly exist). Now (1) is not carefully defined, for it requires $y_{0}, y_{1}$, $y_{2}, \cdots$, and these must be non-negative and sum to unity. The linearization of $(1)$ with multiplication by a power of $r$ (including $r^{0}$ ) leads to a required set of equations to determine the parameters in $P_{s}(r)$ and $Q_{t}(r)$ in terms of $y_{0}$ in general. We then have an equation for $y_{0}$, for determining $y_{0}$ assuming there is a solution.

The corresponding case of a doubly infinite set of probabilities $\left(y_{0}, y_{ \pm 1}, y_{ \pm 2}, \ldots\right)$ is treated in a similar fashion and is sometimes referred to as the Type IV case. We show here that potential solutions are easily set up, given a set of moments and assuming that $y_{0} \neq 0$; transfers of the origin may take care of this case.

We found the studies of Ord quite illuminating and reference may be made to his [5], [6], and [8] papers. There is also his book [7] on frequency distributions with many references to generalizations.

Of course it would be amiss to omit the name of Karl Pearson [9] to whom the basic notion is usually attributed.

\section{BASIC FORMULAE FOR THE SEMI-INFINITE CASE}

\subsection{Development of Formulae}

Let the model be

$$
\begin{gathered}
y_{r}=\left(1+\frac{\alpha-r}{C_{0}+C_{1} x+C_{2} x^{2}}\right) y_{r-1}=k_{r} y_{r-1}, \\
\left(r=1,2, \cdots ; x=r-\mu_{1}^{\prime} ; \mu_{1}^{\prime}=E(r)\right)
\end{gathered}
$$

subject to

$$
y_{0}+k_{1} y_{0}+k_{2} k_{1} y_{0}+\cdots=1 \text {. }
$$

It is assumed that $k_{s} \geq 0, s=1,2, \cdots$. The form in (2) is similar to that used by Ord [5], [6], [8]. Our interest is fitting (2) to statistical data defined as frequencies $n_{0}, n_{1}$, $\ldots ; n_{s}$ being the frequency in the $s$ th cell. The approach is computer oriented; it is 
readily generalized.

The four parameters can be determined by moments using

$$
\sum_{r=1}^{\infty}\left(C_{0}+C_{1} x+C_{2} x^{2}\right)\left(y_{r}-y_{r-1}\right) x^{s}=\sum_{r=1}^{\infty} x^{s}(\alpha-r) y_{r-1}, \quad(s=0,1,2,3)
$$

The right hand component may be defined in terms of $\left(\alpha-\mu_{1}^{\prime}-x\right)$. Basic elements are;

(i) $\sum_{r=1}^{\infty} x^{s}\left(y_{r}-y_{r-1}\right)=\sum_{r=0}^{\infty} x^{s} y_{r}-\left(-\mu_{1}^{\prime}\right)^{s} y_{0}-\sum_{r=1}^{\infty}\left(r-1-\mu_{1}^{\prime}+\mu_{1}^{\prime}\right)^{s} y_{r-1}$

$$
\begin{aligned}
& =\mu_{s}-\left(-\mu_{1}^{\prime}\right)^{s} y_{0}-\left\{\mu_{s}+\left(\begin{array}{l}
s \\
1
\end{array}\right) \mu_{1}^{\prime} \mu_{s-1}+\cdots+\left(\begin{array}{l}
s \\
s
\end{array}\right) \mu_{1}^{\prime s}\right\} \\
& =-\left(-\mu_{1}^{\prime}\right)^{s} y_{0}-\nu_{s}
\end{aligned}
$$

in terms of the mean and central moments, where

$$
\nu_{s}=E(x+1)^{s}-E(x)^{s} \text {. }
$$

For example, $\nu_{0}=0, \nu_{1}=1, \nu_{2}=1, \nu_{3}=3 \mu_{2}+1$.

$$
\text { (ii) } \sum_{r=1}^{\infty} x^{s} y_{r-1}=\sum_{r=1}^{\infty}(\overline{x-1}+1)^{s} y_{r-1}=\mu_{s}+\left(\begin{array}{l}
s \\
1
\end{array}\right) \mu_{s-1}+\cdots=\lambda_{s} \text {. }
$$

For example, $\lambda_{0}=1, \lambda_{1}=1, \lambda_{2}=\mu_{2}+1, \lambda_{3}=\mu_{3}+3 \mu_{2}+1$.

The equations now appear as :

$$
C_{0}\left(\nu_{s}+k_{s} y_{0}\right)+C_{1}\left(\nu_{s+1}+k_{s+1} y_{0}\right)+C_{2}\left(\nu_{s+2}+k_{s+2} y_{0}\right)+C_{3} \lambda_{s}=\lambda_{s+1}
$$

where $k_{s}=\left(-\mu_{1}^{\prime}\right)^{s}, C_{3}=\alpha-\mu_{1}^{\prime}$ and $s=0,1,2,3$. By elementary operations on (5), we find

$$
C_{0} y_{0}+\left(1-y_{0} \mu_{1}^{\prime}\right) C_{1}+\left(1+y_{0} \mu_{1}^{\prime 2}\right) C_{2}+C_{3}=1
$$

and the remaining three equations, in matrix form

$$
\underline{M}\left[C_{1}, C_{2}, C_{3}\right]^{\prime}=\left[h_{1}, h_{2}, h_{3}\right]^{\prime}-C_{0}\left[1, \nu_{1}+\mu_{1}^{\prime}, \nu_{2}+\mu_{1}^{\prime}\right]^{\prime}
$$

where

$$
\underline{M}=\left[\begin{array}{ccc}
\nu_{1}+\mu_{1}^{\prime} & \nu_{2}+\mu_{1}^{\prime} \nu_{1} & \lambda_{1}+\mu_{1}^{\prime} \lambda_{0} \\
\nu_{2}+\mu_{1}^{\prime} \nu_{1} & \nu_{3}+\mu_{1}^{\prime} \nu_{2} & \lambda_{2}+\mu_{1}^{\prime} \lambda_{1} \\
\nu_{3}+\mu_{1}^{\prime} \nu_{2} & \nu_{4}+\mu_{1}^{\prime} \nu_{3} & \lambda_{3}+\mu_{1}^{\prime} \lambda_{2}
\end{array}\right]
$$

and

$$
h_{1}=\lambda_{2}+\mu_{1}^{\prime} \lambda_{1}, h_{2}=\lambda_{3}+\mu_{1}^{\prime} \lambda_{2}, h_{3}=\lambda_{4}+\mu_{1}^{\prime} \lambda_{3} .
$$

From (7), $\left[C_{1}, C_{2}, C_{3}\right]^{\prime}$ is used in (6) to determines $C_{0}$, and a return to (7) to determine $C_{1}, C_{2}$, and $C_{3}$. Then $y_{0}$ and subsequent probabilities follow from (2). 


\subsection{Examples}

\subsubsection{Poisson distribution}

Poisson distributions are those with $y_{r}=e^{-\theta} \theta^{r} / r$ ! For this $C_{0}=\theta, C_{1}=1, C_{2}=0$, and $C_{3}=0$. Once $y_{0}$ is evaluated accurately, then subsequent $y_{r}$ turn out to be all exact Poisson probabilities. For example,

\begin{tabular}{|cccccc|}
\hline \multicolumn{5}{c|}{$y_{r}$} \\
$r$ & 0 & 1 & 2 & 3 & 4 \\
\hline$\theta=0.25$ & 0.7788 & 0.1947 & 0.0243 & 0.0020 & 0.0001 \\
$\theta=0.5$ & 0.6065 & 0.3033 & 0.0758 & 0.0126 & 0.0016 \\
$\theta=0.75$ & 0.4724 & 0.3543 & 0.1329 & 0.0332 & 0.0062 \\
\hline
\end{tabular}

\subsubsection{Stuttering Poisson Distribution}

Stuttering Poisson distributions are those with pr sbability generation function $e^{-a-b-c+a t+b r^{\circ}+c t^{3}}$; we restrict attention here to $a=1, b=1 / 2, c=1 / 3$. The moments are $\mu_{1}^{\prime}=3, \mu_{2}=6, \mu_{3}=14$, and $\mu_{4}=144$. The solutions are

$$
\left\{\begin{array}{l}
C_{0}=\left(-2.476+14.35 y_{0}\right) / \Delta \\
C_{1}=\left(-0.6993+4.421 y_{0}\right) / \Delta \\
C_{2}=\left(0.01748-0.1206 y_{0}\right) / \Delta \\
C_{3}=\left(0.3217-1.904 y_{0}\right) / \Delta \\
\Delta=-0.3601+2.177 y_{0}
\end{array}\right.
$$

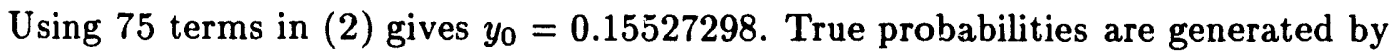

$$
P_{r+1}=\frac{1}{r+1}\left\{a P_{r}+2 b P_{r-1}+3 c P_{r-2}\right\} . \quad\left(r=0,1,2, \cdots ; P_{r}=0 \quad r<0\right)
$$

\begin{tabular}{|ccc|}
\hline$r$ & Discrete Model & True Value \\
\hline 0 & 0.1553 & 0.1599 \\
1 & 0.1678 & 0.1599 \\
2 & 0.1650 & 0.1599 \\
3 & 0.1476 & 0.1599 \\
4 & 0.1204 & 0.1199 \\
5 & 0.0899 & 0.0879 \\
6 & 0.0619 & 0.0613 \\
7 & 0.0396 & 0.0384 \\
8 & 0.0238 & 0.0235 \\
9 & 0.0135 & 0.0137 \\
10 & 0.0074 & 0.0075 \\
11 & 0.0039 & 0.0041 \\
12 & 0.0020 & $0.0 \mathrm{C} 21$ \\
\hline
\end{tabular}




\subsubsection{Binomial distribution}

Binomial distributions are those with probability $\left(\begin{array}{l}n \\ r\end{array}\right) p^{r}(1-p)^{n-r}$; here we examine the case $n=10$. Here $C_{0}=n p q, C_{1}=q, C_{2}=0, C_{3}=p$, and frequencies

\begin{tabular}{|cccccccccccc|}
\hline$r$ & 0 & 1 & 2 & 3 & 4 & 5 & 6 & 7 & 8 & 9 & 10 \\
\hline$p=0.25$ & 0.0563 & 0.1877 & 0.2816 & 0.2503 & 0.1460 & 0.0584 & 0.0162 & 0.0031 & 0.0004 & 0.0000 & 0.0000 \\
$p=0.5$ & 0.0010 & 0.0093 & 0.0440 & 0.1172 & 0.2051 & 0.2461 & 0.2051 & 0.1172 & 0.0440 & 0.0093 & 0.0010 \\
$p=0.75$ & 0.0000 & 0.0000 & 0.0004 & 0.0031 & 0.0162 & 0.0584 & 0.1460 & 0.2503 & 0.2816 & 0.1877 & 0.0563 \\
\hline
\end{tabular}

The recursion is $y_{r}=\{p(n-r+1) /(q r)\} y_{r-1}$, and the derived probabilities are correct.

\subsubsection{Ord's Example of Type I Distribution}

Using a model noted by Ord [5]

$$
\begin{gathered}
y_{r}=\left(1+\frac{C_{2}-x}{C_{0}+C_{1} x}\right) y_{r-1} \\
\left(r=1,2,3, \cdots, \infty ; x=r-\mu_{1}^{\prime} ; C_{2}=\alpha-\mu_{1}^{\prime}\right)
\end{gathered}
$$

and moments $\mu_{1}^{\prime}, \mu_{2}, \mu_{3}$ gives the equations

$$
\left\{\begin{array}{l}
C_{0} y_{0}+C_{1}\left(1-\mu_{1}^{\prime} y_{0}\right)+C_{2}=1 \\
C_{0}+C_{1}\left(1+\mu_{1}^{\prime}\right)+C_{2}\left(1+\mu_{1}^{\prime}\right)=\mu_{2}+\mu_{1}^{\prime}+1 \\
C_{0}\left(1+\mu_{1}^{\prime}\right)+C_{1}\left(3 \mu_{2}+1+\mu_{1}^{\prime}\right)+C_{2}\left(1+\mu_{2}+\mu_{1}^{\prime}\right)=1+3 \mu_{2}+\mu_{3}+\mu_{2} \mu_{1}^{\prime}+\mu_{1}^{\prime}
\end{array}\right.
$$

and solutions, when $\mu_{3}=0$

$$
\left\{\begin{array}{l}
C_{0}=\mu_{2}\left\{2+\mu_{1}^{\prime} y_{0}\left[1-\left(1+\mu_{1}^{\prime}\right)^{2} / \mu_{2}\right]\right\} / \Delta \\
C_{1}=\left\{1+y_{0}\left[\mu_{2}-\left(1+\mu_{1}^{\prime}\right)^{2}\right]\right\} / \Delta \\
C_{2}=\left\{1-y_{0}\left[3 \mu_{2}-\mu_{1}^{\prime 2}-2 \mu_{1}^{\prime}+1+\mu_{1}^{\prime 2}\left(1+\mu_{1}^{\prime}\right) / \mu_{2}\right]\right\} / \Delta \\
\Delta=2-y_{0}\left[2+\mu_{1}^{\prime}+\mu_{1}^{\prime 2}\left(1+\mu_{1}^{\prime}\right) / \mu_{2}\right]
\end{array}\right.
$$

As an exar.ple, take the triangular distribution

$$
y_{r}=\frac{r+1}{(n+1)^{2}}, \quad(r=0,1, \cdots, n), \quad y_{r}=\frac{2 n-r+1}{(n+1)^{2}} \cdot(r=n+1, n+2, \cdots, 2 n)
$$

with moments

$$
\mu_{1}^{\prime}=n, \quad \mu_{2}=n(n+2) / 6, \quad \mu_{3}=0 .
$$

For $n=4$, 


\begin{tabular}{|ccccc|}
\hline$r$ & True Value & 1 & 2 & 3 \\
\hline 0 & 0.0400 & 0.0432 & 0.0425 & 0.0495 \\
1 & 0.0800 & 0.0774 & 0.0766 & 0.0742 \\
2 & 0.1200 & 0.1211 & 0.1204 & 0.1096 \\
3 & 0.1600 & 0.1632 & 0.1626 & 0.1535 \\
4 & 0.2000 & 0.1861 & 0.1852 & 0.1910 \\
5 & 0.1600 & 0.1750 & 0.1739 & 0.1910 \\
6 & 0.1200 & 0.1311 & 0.1300 & 0.1363 \\
7 & 0.0800 & 0.0742 & 0.0734 & 0.0644 \\
8 & 0.0400 & 0.0289 & 0.0287 & 0.0215 \\
9 & - & - & 0.0064 & 0.0062 \\
10 & - & - & 0.0004 & 0.0018 \\
11 & - & - & -0.0000 & 0.0006 \\
12 & - & - & 0.0000 & 0.0002 \\
13 & - & - & - & 0.0001 \\
14 & - & - & - & 0.0000 \\
\hline$\mu_{1}^{\prime}$ & 4.0 & 3.9649 & 3.9999 & 3.9999 \\
$\mu_{2}$ & 4.0 & 3.8616 & 4.0001 & 3.9977 \\
$\sqrt{\beta_{1}}$ & 0 & -0.0661 & 0.0003 & -0.0094 \\
$\beta_{2}$ & 1.77 & 2.3460 & 2.4493 & 2.8069 \\
\hline
\end{tabular}

The basic series for $y_{0}$ was taken to 9,13 , and 21 terms in computing the probabilities in columns 1, 2, and 3. For the parameters,

$$
\left\{\begin{array}{l}
C_{0}=\left(32-336 y_{0}\right) / \Delta \\
C_{1}=\left(4-84 y_{0}\right) / \Delta \\
C_{2}=\left(4-36 y_{0}\right) / \Delta \\
\Delta=8-104 y_{0}
\end{array}\right.
$$

\section{BASIC FORMULAE FOR THE BOUNUED CASE}

\subsection{Development of Formulae}

Let the model be

$$
\begin{gathered}
y_{r}=\left(1+\frac{C_{3}-x}{C_{0}+C_{1} x+C_{2} x^{2}}\right) y_{r-1}=k_{r} y_{r-1}, \\
\left(r=1,2, \cdots N ; x=r-\mu_{1}^{\prime} ; \mu_{1}^{\prime}=E(r)\right)
\end{gathered}
$$

subject to

$$
y_{0}+k_{1} y_{0}+k_{2} k_{1} y_{0}+\cdots+k_{N} k_{N-1} \cdots k_{3} k_{2} k_{1} y_{0}=1
$$

and

$$
y_{N}=k_{N}-k_{1} y_{0}
$$


It is assumed that $k_{s} \geq 0, s=1,2, \cdots, N$. We use

$$
\begin{gathered}
\sum_{r=1}^{N} y_{r} x^{s}=\mu_{s}-\left(-\mu_{1}^{\prime}\right)^{s} y_{0} \\
\sum_{r=1}^{N} y_{r-1} x^{s}=\lambda_{s}-T^{s} y_{N} \quad\left(T=N-\mu_{1}^{\prime}+1, \lambda_{s}=E(x+1)^{s}\right) \\
\sum_{r=1}^{N}\left(y_{r}-y_{r-1}\right) x^{s}=-\nu_{s}-\left(-\mu_{1}^{\prime}\right)^{s} y_{0}+T^{s} y_{N} \quad\left(\nu_{s}=E(x+1)^{s}-E\left(x^{s}\right)\right)
\end{gathered}
$$

The six parameters can be determined by moments using

$$
\begin{aligned}
\left(y_{r}-3_{r-1}\right)\left(C_{0}+C_{1} x+C_{2} x^{2}\right) & =\left(C_{3}-x\right) y_{r-1} \\
C_{0}\left(-y_{0}+y_{N}\right)+C_{1}\left(-\nu_{1}+\mu_{1}^{\prime} y_{0}+T y_{N}\right) & +C_{2}\left(-\nu_{2}-\mu_{1}^{\prime 2} y_{0}+T^{2} y_{N}\right) \\
& -C_{3}\left(\lambda_{0}-y_{N}\right)=-\left(\lambda_{1}-T y_{N}\right) \\
& \\
C_{0}\left(-\nu_{1}+\mu_{1}^{\prime} y_{0}+T y_{N}\right): C_{1}\left(-1 / 2-\mu_{1}^{\prime 2} y_{0}\right. & \left.+T^{2} y_{N}\right)+C_{2}\left(-\nu_{3}+\mu_{1}^{\prime 3} y_{0}+T^{3} y_{N}\right) \\
& -C_{3}\left(\lambda_{1}-T y_{N}\right)=-\left(\lambda_{2}-T^{2} y_{N}\right) \\
& \\
C_{0}\left(-\nu_{2}-\mu_{1}^{\prime 2} y_{0}+T^{2} y_{N}\right)+C_{1}\left(-\nu_{3}+\mu_{1}^{\prime 3} y_{0}\right. & \left.+T^{3} y_{N}\right)+C_{2}\left(-\nu_{4}-\mu_{1}^{\prime 4} y_{0}+T^{4} y_{N}\right) \\
& -C_{3}\left(\lambda_{2}-T^{2} y_{N}\right)=-\left(\lambda_{3}-T^{3} y_{N}\right) \\
& \\
C_{0}\left(-\nu_{3}+\mu_{1}^{\prime 3} y_{0}+T^{3} y_{N}\right)+C_{1}\left(-\nu_{4}-\mu_{1}^{\prime 4} y_{0}\right. & \left.+T^{4} y_{N}\right)+C_{2}\left(-\nu_{5}+\mu_{1}^{\prime 5} y_{0}+T^{5} y_{N}\right) \\
& -C_{3}\left(\lambda_{3}-T^{3} y_{N}\right)=-\left(\lambda_{4}-T^{4} y_{N}\right) .
\end{aligned}
$$

From the last three equations, set up $C_{0}, C_{1}, C_{2}$ in terms of $C_{3}$, insert these derived forms in the first equation and thus find $C_{0}, C_{1}, C_{2}$, and $C_{3}$ in terms of $y_{0}$ and $y_{N}$. Then $y_{0}+k_{1} y_{0}+\cdots=1$ or $y_{0}=1 /\left(1+k_{1}+k_{2} k_{1}+\cdots+k_{N} k_{N-1} \cdots k_{1}\right)$ and $k_{N} k_{N-1} k_{N-2} \cdots k_{1} y_{0}=y_{N}$.

If the model is

$$
y_{r}=\left(1+\frac{C_{2}-x}{C_{0}+C_{1} x}\right) y_{r-1}
$$

then the gerieral solutions are, assuming existence

$$
\left\{\begin{array}{l}
C_{0}=\left(-2 \mu_{2}^{2}+y_{0} A_{1}+y_{N} A_{2}+y_{0} y_{N} A_{3}\right) / \Delta \\
C_{1}=\left(-\mu_{2}-\mu_{3}+y_{0} B_{1}+y_{N} B_{2}+y_{0} y_{N} B_{3}\right) / \Delta \\
C_{2}=\left(-\mu_{2}+\mu_{3}+y_{0} D_{1}+y_{N} D_{2}+y_{0} y_{N} D_{3}\right) / \Delta \\
\Delta=-2 \mu_{2}+y_{0} E_{1}+y_{N} E_{2}+y_{0} y_{N} E_{3}
\end{array}\right.
$$


where

$$
\begin{aligned}
A_{1} & =\mu_{1}^{\prime}\left(\mu_{2}+2 \mu_{1}^{\prime} \mu_{2}+\mu_{3}+\mu_{1}^{\prime} \mu_{3}+\mu_{1}^{\prime 2} \mu_{2}-\mu_{2}^{2}\right) \\
A_{2} & =\mu_{1}^{\prime}\left(\mu_{2}-\mu_{1}^{\prime} \mu_{2}-2 \mu_{3}-\mu_{1}^{\prime} \mu_{3}-\mu_{1}^{\prime 2} \mu_{2}+\mu_{2}^{2}\right)-\mu_{3}+3 \mu_{2}^{2}+\mu_{2} \\
& +m\left(-\mu_{2}+2 \mu_{3}+2 \mu_{1}^{\prime} \mu_{2}-\mu_{2}^{2}+2 \mu_{1}^{\prime} \mu_{3}+3 \mu_{1}^{\prime 2} \mu_{2}\right) \\
& -m^{2}\left(\mu_{2}+\mu_{3}+3 \mu_{1}^{\prime} \mu_{2}\right)+m^{3} \mu_{2} \\
A_{3} & =-\mu_{1}^{\prime}\left\{m(m-1)^{2}+m\left[-3\left(\mu_{2}+\mu_{1}^{\prime}+\mu_{1}^{\prime 2}+\mu_{1}^{\prime} \mu_{2}\right)-\mu_{3}-\mu_{1}^{\prime 3}\right]\right. \\
& \left.+m^{2}\left(4 \mu_{1}^{\prime}+2 \mu_{2}+2 \mu_{1}^{\prime 2}\right)-m^{3} \mu_{1}^{\prime}\right\} \\
B_{1} & =\mu_{2}+2 \mu_{1}^{\prime} \mu_{2}+\mu_{1}^{\prime 2} \mu_{2}+\mu_{1}^{\prime} \mu_{3}-\mu_{2}^{2}+\mu_{3} \\
B_{2} & =m(m-1)^{2}-\mu_{1}^{\prime}\left(1+2 \mu_{1}^{\prime}+\mu_{2}+\mu_{1}^{\prime 2}+\mu_{1}^{\prime} \mu_{2}+\mu_{3}\right)+\mu_{2}\left(1+\mu_{2}\right) \\
& +m\left(4 \mu_{1}^{\prime}+\mu_{2}+3 \mu_{1}^{\prime 2}+\mu_{3}+2 \mu_{1}^{\prime} \mu_{2}\right)-m^{2}\left(3 \mu_{1}^{\prime}+\mu_{2}\right) \\
B_{3} & =-m(m-1)^{2}-m\left(3 \mu_{1}^{\prime}\left(1+\mu_{2}+\mu_{1}^{\prime}\right)+\mu_{1}^{\prime 3}+3 \mu_{2}+\mu_{3}\right) \\
& +m^{2}\left(4 \mu_{1}^{\prime}+2 \mu_{1}^{\prime 2}+2 \mu_{2}\right)-m^{3} \mu_{1}^{\prime} \\
D_{1} & =\mu_{1}^{\prime}\left(\mu_{1}^{\prime}+\mu_{1}^{\prime 2}-2 \mu_{2}-\mu_{1}^{\prime} \mu_{2}-2 \mu_{3}\right)+\mu_{2}+3 \mu_{2}^{2}-\mu_{3} \\
D_{2} & =-\mu_{2}\left[1+\mu_{1}^{\prime}\left(1-\mu_{1}^{\prime}\right)+3 \mu_{2}\right]+\mu_{3}+2 \mu_{1}^{\prime} \mu_{3}+m\left(\mu_{2}-2 \mu_{3}-2 \mu_{1}^{\prime} \mu_{2}\right)+m^{2} \mu_{2} \\
D_{3} & =m \mu_{1}^{\prime}\left[1+3\left(\mu_{2}+\mu_{1}^{\prime}+\mu_{1}^{\prime 2}\right)\right]+m^{2}\left(-2 \mu_{1}^{\prime}-4 \mu_{1}^{\prime 2}+2 \mu_{1}^{\prime} \mu_{2}+\mu_{3}\right)+m^{3}\left(\mu_{1}^{\prime}-\mu_{2}\right) \\
E_{1} & =\mu_{1}^{\prime}\left(\mu_{1}^{\prime}+\mu_{1}^{\prime 2}+\mu_{2}\right)+2 \mu_{2} \\
E_{2} & =m(m-1)^{2}-\mu_{1}^{\prime}\left[\mu_{2}+\left(1+\mu_{1}^{\prime}\right)^{2}\right]+\mu_{2}+m\left[\mu_{1}^{\prime}\left(4+3 \mu_{1}^{\prime}\right)+\mu_{2}\right]-3 m^{2} \mu_{1}^{\prime} \\
E_{3} & =-m(m-1)^{2}-3 m\left[\mu_{1}^{\prime}\left(1+\mu_{1}^{\prime}\right)+\mu_{2}\right]+m^{2}\left[\mu_{1}^{\prime}\left(4+\mu_{1}^{\prime}\right)+\mu_{2}\right]-m^{3} \mu_{1}^{\prime}
\end{aligned}
$$

and $m=N+1$.

\subsection{Examples}

\subsubsection{Triangular Distribution}

For the discrete triangular distribution of 2.2.4. with $N=8, \mu_{1}^{\prime}=4, \mu_{2}=4$, and $\mu_{3}=0$ using the fundamental equations

$$
\left\{\begin{array}{l}
C_{0}\left(-y_{0}+y_{8}\right)+C_{\mathrm{i}}\left(-1+4 y_{0}+5 y_{8}\right)+C_{2}\left(y_{8}-1\right)=-1+5 y_{8} \\
C_{0}\left(-1+9 y_{0}\right)+C_{1}\left(4-36 y_{0}\right)+4 C_{2}=0 \\
C_{0}\left(4-36 y_{0}\right)+\left(-8+144 y_{0}\right)=12
\end{array}\right.
$$

we find solutions

$$
\left\{\begin{array}{l}
C_{0}=\left[8-84 y_{0}+y_{8}\left(-88+612 y_{0}\right)\right] / \Delta \\
C_{1}=\left[1-21 y_{0}+y_{8}\left(-5+153 y_{0}\right)\right] / \Delta \\
C_{2}=\left[1-9 y_{0}+y_{8}\left(-17+153 y_{0}\right)\right] / \Delta \\
\Delta=2-26 y_{0}+y_{8}\left(-26+306 y_{0}\right) \\
k_{r}=1+\left(C_{2}-x\right) /\left(C_{0}+C_{1} x\right) . \quad(x=r-4, r=1,2, \cdots, 8) .
\end{array}\right.
$$


If we assume the true probabilities in the parameters, then

$$
C_{0}=5.125, \quad C_{1}=0.5, \quad C_{2}=0.5, \quad \Delta=0.4096 .
$$

We use our scheme for $y_{0}$ and $y_{N}(N=8)$ iteratively and we find the 15 th iteratives to be $y_{0}=0.0394944261$, and $y_{8}=0.0394944261$ with the recursive parameters

$$
C_{0}=5.102939, \quad C_{1}=0.500000, \quad C_{2}=0.500000, \quad \Delta=0.423591678 .
$$

The evaluated probabilities (symmetric to 6 significant figures) are,

\begin{tabular}{|cccccc|}
\hline$r$ & 0 and 8 & 1 and 7 & 2 and 6 & 3 and 5 & 4 \\
\hline$y_{r}$ & 0.039494 & 0.07786 & 0.125302 & 0.166136 & 0.182414 \\
True & 0.040000 & 0.08000 & 0.120000 & 0.160000 & 0.200000 \\
\hline
\end{tabular}

and computed moments

$$
\mu_{1}^{\prime}=4.000000, \quad \mu_{2}=4.000000, \quad \mu_{3}=-0.000001 .
$$

From numerical evidence it appears that $k_{5} k_{4}=k_{6} k_{3}=k_{7} k_{2}=k_{8} k_{1}=k_{9} k_{0}$. From the formulas for $C_{0}, C_{1}, C_{2}$ we find this leads to $C_{1}=1 / 2, C_{2}=1 / 2$ and $y_{0}=y_{8}$. Hence

$$
k_{r}=\frac{2 C_{0}+5-r}{2 C_{0}+r-4} \quad(r=0,1, \cdots, 8)
$$

and

$$
C_{0}=\frac{8-172 y_{0}+612 y_{0}^{2}}{2-52 y_{0}+306 y_{0}^{2}}
$$

The equation for $y_{0}$ is

$$
y_{0}\left(2+k_{1}+2 k_{1} k_{2}+2 k_{1} k_{2} k_{3}+k_{1} k_{2} k_{3} k_{4}\right)=1 .
$$

Using it iteratively with $y_{0}=0.04$ initially, the 14 th iterate is $y_{0}=0.039494425$.

\subsubsection{Non-Symmetric Distribution}

Consider another example with $\mu_{1}^{\prime}=2.2, \mu_{2}=1.56$ and $\mu_{3}=-0.144$, which has probabilities

$$
y_{0}=1 / 10, y_{1}=2 / 10, \quad y_{2}=3 / 10, \quad y_{3}=2 / 10, y_{4}=2 / 10
$$

The model is

$$
y_{r}=\left(1+\frac{C_{2}-x}{C_{0}+C_{1} x}\right) y_{r-1} \quad(r=1,2,3,4 ; x=r-2.2)
$$


and given the moments, solutions are

$$
\left\{\begin{array}{l}
C_{0}=4.8672-28.776 y_{0}-y_{4}\left(20.16-105.6 y_{0}\right) / \Delta \\
C_{1}=1.4160-13.080 y_{0}-y_{4}\left(4.80-48.0 y_{0}\right) / \Delta \\
C_{2}=1.7040-10.712 y_{0}-y_{4}\left(8.40-51.2 y_{0}\right) / \Delta \\
\Delta=3.1200-22.040 y_{0}-y_{4}\left(15.0-104.0 y_{0}\right)
\end{array}\right.
$$

After 107 iterations, we have $y_{0}=0.09276122$, and $y_{4}=0.19184435$, with computed moments, 2.2001, 1.5596, and -C.1498.

\subsubsection{Truncated Poisson Distribution}

The truncated Poisson distribution may be considered as a doubly bounded distribution

$$
P_{r}=k_{N}^{*} \frac{\theta^{r}}{r !}, \quad(r=0,1,2, \cdots, N, \theta>0)
$$

and $k_{N}^{*}=\left(1+\theta / 1 !+\cdots+\theta^{N} / N !\right)^{-1}, P_{0}=k_{N}^{*}, P_{N}=k_{N}^{*} \theta^{N} / N !$. Recurrence is

$$
y_{r}=\left(1+\frac{C_{2}-x}{C_{0}+C_{1} x}\right) y_{r-1} \quad\left(r=1,2, \cdots, N ; x=r-\mu_{1}^{\prime}\right)
$$

where $C_{0}=\mu_{1}^{\prime}, C_{1}=1, C_{2}=\theta-\mu_{1}^{\prime}$, and $\mu_{1}^{\prime}=\theta\left(1-k_{N}^{*} \theta^{N} / N !\right)$.

For another example, we take $P_{s}=k^{*} \theta^{s+2} /(s+2) !, \quad(s=0,1,2,3,4)$, with $\theta=1$. The moments are $\mu_{1}^{\prime}=0.390716, \mu_{2}=0.446954, \mu_{3}=0.549920$, and $\mu_{4}=1.303602$. We find $C_{0}=2.390716, C_{1}=1$, and $C_{2}=-1.390716$, and computed $y_{s}$ identical to the true probabilities $P_{s}$.

\section{THE DOUBLY INFINITE CASE}

\subsection{Formulae}

Suppose the probability $y_{r}$ now includes the negative integers and $r=0, \pm 1, \pm 2, \cdots$. Then

$$
\begin{gathered}
\sum_{r=-\infty}^{\infty} x^{s}\left(y_{r}-y_{r-1}\right)=-\nu_{s} \\
\sum_{r=-\infty}^{\infty} x^{s} y_{r-1}=\lambda_{s} .
\end{gathered}
$$

In other words the new equations for $\left(C_{0}, C_{1}, C_{2}, C_{3}\right)$ are those in (5) with $y_{0}$ taken to be zero (this does not mean that the actual $y_{0}$ is zero). We have after elementary operations,

$$
\left\{\begin{array}{l}
C_{1}+C_{2}+C_{3}=1 \\
C_{0}+3 \mu_{2} C_{2}=\mu_{2} \\
3 \mu_{2} C_{1}+\left(4 \mu_{3}+3 \mu_{2}\right) C_{2}+\mu_{2} C_{3}=\mu_{3}+2 \mu_{2}, \\
3 \mu_{2} C_{0}+\left(\mu_{3}+3 \mu_{2}\right) C_{1}+\left(5 \mu_{4}+6 \mu_{3}+4 \mu_{2}\right) C_{2}+\left(2 \mu_{2}+\mu_{3}\right) C_{3}=\mu_{4}+3 \mu_{3}+3 \mu_{2} .
\end{array}\right.
$$


leading to the solution (if valid)

$$
\left\{\begin{array}{l}
C_{0}=\mu_{2}\left(4 \beta_{2}-3 \beta_{1}-1 / \mu_{2}\right) / \Delta \\
C_{1}+\alpha=\mu_{1}^{\prime}+\left(8 \beta_{2}-9 \beta_{1}-12+1 / \mu_{2}\right) / \Delta \\
C_{2}=\left(2 \beta_{2}-3 \beta_{1}-6+1 / \mu_{2}\right) / \Delta \\
\alpha=\mu_{1}^{\prime}+1 / 2-\left(\mu_{3} / \mu_{2}\right)\left(\beta_{2}+3-1 / \mu_{2}\right) / \Delta \\
\Delta=10 \beta_{2}-12 \beta_{1}-18+2 / \mu_{2}
\end{array}\right.
$$

with $\sqrt{\beta_{1}}=\mu_{3} / \mu_{2}^{3 / 2}$ and $\beta_{2}=\mu_{4} / \mu_{2}^{2}$. We have then

$$
y_{0}=\left\{1+\left(k_{1}+k_{0}^{-1}\right)+\left(k_{2} k_{1}+k_{-1}^{-1} k_{0}^{-1}\right)+\left(k_{3} k_{2} k_{1}+k_{-2}^{-1} k_{-1}^{-1} k_{0}^{-1}\right)+\cdots\right\}^{-1}
$$

involving in some cases a slowly converging series.

\subsection{Examples}

\subsubsection{Pearson Type IV Moments}

Consider the case, $\mu_{1}^{\prime}=1, \mu_{2}=2, \sqrt{\beta}_{1}=1.5, \beta_{2}=12$. Using (8)

$$
C_{0}=1.072368, \quad C_{1}=0.750120, \quad C_{2}=0.154605, \quad C_{3}=0.095274,
$$

and using 75 terms of (9), the returned computed moments are

$$
\mu_{1}^{\prime}=0.999998, \quad \mu_{2}=1.999805, \sqrt{\beta_{1}}=1.4930, \quad \beta_{2}=11.3460 .
$$

\subsubsection{Discrete Pseudo-Normal Distribution}

Next consider the discrete pseudo-normal distribution, using the moments for the continuous case, $\mu_{1}^{\prime}=0, \mu_{2}=1, \sqrt{\beta_{1}}=0, \beta_{2}=3$. Here $y_{0}=0.3974$ ( using 100 or so terms of $(9))$ and $1 / \sqrt{2 \pi}=0.3989$. Moreover $y_{1} / y_{0}=0.6112$ and $e^{-1 / 2}=0.6065$. Using the probabilities from the recursion, the first four moment parameters check to at least five significant digits and

$$
y_{r}=\left\{\frac{(r-4)^{2}+2}{(r+3)^{2}+2}\right\} y_{r-1} . \quad(r=0, \pm 1, \cdots)
$$

For a general normal case, with $\mu_{1}^{\prime}=\mu, \mu_{2}=\sigma^{2}, \sqrt{\beta_{1}}=0, \beta_{2}=3$, we find

$$
y_{r}=\left\{\frac{x^{2}-\left(6 \sigma^{2}+2\right) x+12 \sigma^{4}+5 \sigma^{2}+1}{x^{2}+6 \sigma^{2} x+\left(12 \sigma^{2}-1\right) \sigma^{2}}\right\} y_{r-1} . \quad\left(r=0, \pm 1, \ldots ; x=r-\mu_{1}^{\prime}\right) .
$$

Now define

$$
y_{r}=k e^{-r^{2} / 2}, \quad(r=0, \pm 1, \cdots)
$$


Then direct calculation finds $\mu_{2}=0.999999788, \mu_{4}=3.00007069$. Using these moments, we retrieve $y_{0}, y_{ \pm 1}$, etc. and the recurrence

$$
y_{r}=\left(\frac{11.9980855-7.999362604 r+r^{2}}{10.9987+5.999362608 r+r^{2}}\right) y_{r-1} \quad(r=0, \pm 1, \cdots),
$$

with $y_{0}$ differing slightly from 0.3974 found by taking $\mu_{2}=1, \mu_{4}=3\left(\mu_{1}^{\prime}=0, \mu_{3}=0\right)$.

\subsubsection{Pearson Type VII Moments}

Suppose a distribution has moments

$$
\mu_{1}^{\prime}=0, \mu_{2}=3, \mu_{3}=0, \mu_{4}=75 .
$$

The Type IV model yields the results:

$$
y_{r}=\left(\frac{12-4 r+r^{2}}{9+2 r+r^{2}}\right) y_{r-1} . \quad(r=0, \pm 1, \pm 2, \cdots)
$$

The first few values of the probabilities are (there being symmetry)

\begin{tabular}{|cccccccc|}
\hline$r$ & 0 & 1 & 2 & 3 & 4 & 5 & 6 \\
\hline$y$ & 0.2751 & 0.2063 & 0.09709 & 0.03641 & 0.01324 & 0.00512 & 0.00215 \\
\hline
\end{tabular}

Note that $y_{1} / y_{0}=0.75, y_{2} / y_{1}=0.4706, y_{3} / y_{2}=0.375$. The returned moments are

$$
\mu_{1}^{\prime}=0, \mu_{2}=2.9997, \mu_{3}=-0.0008, \mu_{4}=70.2831,
$$

using $y_{-75}$ to $y_{75}$ in the computations. Note that $k_{1-r}=k_{r}^{-1}$.

The fit is poor when the distribution used is $y_{0}=p, y_{ \pm r}=p q^{|r|} / 2(r \neq 0)$, with $p=q=1 / 2$, and $y_{r} / y_{r-1}=1 / 2, r \geq 2$. The example serves as a reminder that some structures will fail in this Type IV and other similar models.

\subsubsection{Bessel Distribution}

Bessel Distribution (see Johnson and Kotz, [3]; Skellam, [10]) can arise as the distribution of the difference between two independent Poisson variables (means $\theta_{1}$ and $\theta_{2}$ ) and probability generating function (P.G.F.); $\exp \left(\theta_{1} t+\theta_{2} / t-\theta_{1}-\theta_{2}\right)$ with recurrence $\theta_{2} P_{s+2}=\theta_{1} P_{s}-(s+1) P_{s+1}$, then

$$
\operatorname{Pr}\left(r_{1}-r_{2}=t\right)=e^{-\theta_{1}-\theta_{2}}\left(\theta_{1} / \theta_{2}\right)^{t / 2} I_{t}\left(2 \sqrt{\theta_{1} \theta_{2}}\right) \quad\left(\theta_{1}, \theta_{2}>0\right)
$$

in terms of the Bessel function $I_{t}(\cdot)$.

The cumulant generating function is

$$
\theta_{1} e^{\alpha}+\theta_{2} e^{-\alpha}-\theta_{1}-\theta_{2}
$$


so that

$$
\left\{\begin{array}{l}
\kappa_{1}=\theta_{1}-\theta_{2}, \\
\kappa_{2}=\theta_{1}+\theta_{2}, \\
\kappa_{3}=\theta_{1}-\theta_{2}, \\
\kappa_{4}=\theta_{1}+\theta_{2} .
\end{array}\right.
$$

and if $\theta_{1}=\theta_{2}=\theta$, then $\mu_{1}^{\prime}=0, \mu_{2}=2 \theta, \mu_{3}=0$, and $\mu_{4}=2 \theta+12 \theta^{2}$. Moreover, in this rase

$$
y_{0}=e^{-2 \theta} I_{0}(2 \theta) \text {. }
$$

If $\theta=1$, then the doubly infinite model

$$
y_{r}=\left(1+\frac{C_{3}}{C_{0}+C_{1}} \frac{-r}{r+C_{2} r^{2}}\right) y_{r-1}, \quad(r=0, \pm 1, \pm 2, \cdots)
$$

with $C_{0}=1.5, C_{1}=5 / 12, C_{2}=1 / 112, C_{3}=1 / 2$ and

$$
y_{r}=\left(\frac{24-7 r+r^{2}}{18+5 r+r^{2}}\right) y_{r-1},
$$

and from $y_{0}=\left(1+2 k_{1}+2 k_{1} k_{2}+\cdots\right)^{-1}$, we find $y_{0}=0.294427$ (true is 0.308508 ).

\begin{tabular}{|ccc|}
\hline$r$ & $\begin{array}{c}\text { Pearson Discrete } \\
\text { Distribution }\end{array}$ & $\begin{array}{c}\text { Bessel } \\
\text { Distribution }\end{array}$ \\
\hline 0 & 0.294427 & 0.308508 \\
1 & 0.220820 & 0.215269 \\
2 & 0.096609 & 0.093239 \\
3 & 0.027603 & 0.028791 \\
4 & 0.006134 & 0.006865 \\
5 & 0.001263 & 0.001330 \\
6 & 0.000271 & 0.000217 \\
7 & 0.000064 & 0.000030 \\
8 & 0.000017 & 0.000004 \\
9 & 0.000005 & \\
10 & 0.000002 & \\
11 & 0.000001 & \\
\hline
\end{tabular}

\subsubsection{Bessel Distribution with Bias}

We have $\theta_{1}=4, \theta_{2}=1$, then, $\mu_{1}^{\prime}=3, \mu_{2}=5, \mu_{3}=3$, and $\mu_{4}=80$, Basic model is

$$
y_{r}=\left(1+\frac{C_{3}-x}{C_{0}+C_{1} x+C_{2} x^{2}}\right) y_{r-1} \quad(r=0, \pm 1, \pm 2, \cdots, x=r-3)
$$

and solutions are $C_{0}=4.574468, C_{1}=0.7375589, C_{2}=0.028369$, and $C_{3}=0.234043$. Pearson estimates $y_{0}$ is 0.076536 and true is 0.076152 . 


\section{A GENERALIZATION}

\subsection{Formulae}

Suppose in the recursive scheme in (2) that the numerator is of degree $t(\geq 1)$ whereas the denominator is of degree $r>t$, both in the variable $x=r-\mu_{1}^{\prime}$. Then $2 r+t-1$ moments will be required, the model being

$$
y_{r}=\left\{1+\frac{\sum_{\lambda=0}^{t} \hat{C}_{\lambda} x^{\lambda}}{\sum_{\lambda=0}^{r} C_{\lambda} x^{\lambda}}\right\} y_{r-1} \quad\left(r=1,2, \cdots ; y_{0} \neq 0\right)
$$

where $\hat{C}_{\lambda}$ and $C_{\lambda}$ are rational fractions with numerator and denominator of degree 1 and linear in $y_{0}$.

The equations become

$$
\begin{array}{cc}
C_{0}\left(\nu_{s}+k_{s} y_{0}\right)+ & C_{1}\left(\nu_{s+1}+k_{s+1} y_{0}\right)+\cdots+C_{r}\left(\nu_{r+s}+k_{r+s} y_{0}\right) \\
+ & \hat{C}_{0} \lambda_{s}+\hat{C}_{1} \lambda_{s+1}+\cdots+\hat{C}_{t} \lambda_{s+t}=0 . \\
& \left(\hat{C}_{1}=-1 ; s=0,1, \cdots, r+t\right)
\end{array}
$$

We consider the case for which the probabilities are zero on the negative real axis.

The $r+t+1$ equations are linear in the unknown $y_{0}(\neq 0)$. By multiplying the $s$ th equation $(s=0,1, \cdots, r+t)$ by $\mu_{1}^{\prime}$ and adding to the $(s+1)$ th equation, there will be one equation only involving $y_{0}$ explicitly, namely when $s=0$. The remaining $r+t$ equations will involve $r+t+1$ unknowns. By a simple matrix inversion, we can find $r+t$ of the parameters $C$ and $\hat{C}$ in terms of an excluded parameter. A return to (10) with $s=0$ determines the $r+t+1$ unknowns.

The type IV case equations follow by taking $y_{0}=0$ in (10) but not in the equation analogous to (9).

\subsection{The Hansmann Distribution}

Hansmann [2] considered the Pearson Type symmetric family defined by

$$
\frac{1}{y} \frac{d y}{d x}=\frac{-x}{C_{0}+C_{2} x^{2}+C_{4} x^{4}} \quad(-\infty<x<\infty)
$$

and gave explicit solutions for seven different forms. A discrete form of this would be $y_{r}=k_{r} y_{r-1}$ with

$$
\begin{gathered}
y_{r}=\left(1+\frac{\alpha-r}{C_{0}+C_{1} x+C_{2} x^{2}+C_{3} x^{3}+C_{4} x^{4}}\right) y_{r-1} \\
\left(r= \pm 1, \pm 2, \cdots ; x=r-\mu_{1}^{\prime}, C_{5}=\alpha-\mu_{1}^{\prime}\right)
\end{gathered}
$$

in the doubly infinite case; recall that here $y_{-1}=y_{0} / k_{0}, y_{-2}=y_{0} /\left(k_{0} k_{-1}\right)$ etc. In (11) there is no contradiction when positive and negative values of the argument are considered, whereas an exact discrete form would clearly be invalid; however it could be considered when $y_{r}$ refers to the positive axis only. In the doubly infinite case, $C_{1}$ 
and $C_{3}$ in (12) play an important role.

There are six parameters in (12) determined from

$$
\begin{aligned}
C_{0} y_{0}+C_{1}\left(\nu_{1}-y_{0} \mu_{1}^{\prime}\right) & +C_{2}\left(\nu_{2}+y_{0} \mu_{1}^{\prime 2}\right)+C_{3}\left(\nu_{3}-y_{0} \mu_{1}^{\prime 3}\right) \\
& +C_{4}\left(\nu_{4}+y_{0} \mu_{1}^{\prime}\right)+C_{5} \lambda_{0}=\lambda_{1}
\end{aligned}
$$

and

$$
\begin{gathered}
C_{0}\left(\nu_{s}+\mu_{1}^{\prime} \nu_{s-1}\right)+C_{1}\left(\nu_{s+1}+\mu_{1}^{\prime} \nu_{s}\right)+C_{2}\left(\nu_{s+2}+\mu_{1}^{\prime} \nu_{s+1}\right) \\
+C_{3}\left(\nu_{s+3}+\mu_{1}^{\prime} \nu_{s+2}\right)+C_{4}\left(\nu_{s+4}+\mu_{1}^{\prime} \nu_{s+3}\right)+C_{5}\left(\lambda_{s}+\mu_{1}^{\prime} \lambda_{s-1}\right)=\lambda_{s+1}+\mu_{1}^{\prime} \lambda_{s} . \\
(s=1,2,3,4,5)
\end{gathered}
$$

These 5 equations (14) in 6 unknowns are used in (13) to determine the 6 unknowns.

For the double infinite case, use the modified parameters given in section 4.

\subsection{Further Comments on the Models}

We can not say what further forms of the fundamental recurrence w'll turn out to be useful for practical situations. Much will depend on sample sizes and the range of $r$ in the probability $y_{r}$; another important factor is the response of the model to sampling errors in higher sample moments (note, for example, that for a quartic denominator and cubic numerator ten moments are required). If we are not dealing with experimental data but with theoretical structures for which a set of moments are available then a model may prove of some use although perhaps difficult to gain insight from.

There are several choices available for the numerator if we extend models like (12). Consider the case of a cubic. Then

$$
k_{r}=\frac{\hat{C}_{0}+\hat{C}_{1} x+\hat{C}_{2} x^{2}+\hat{C}_{3} x^{3}}{C_{0}+C_{1} x+C_{2} x^{2}+C_{3} x^{3}+C_{4} x^{4}}
$$

and one parameter is available for disposal. The basic form in (1) and its extension in (12) would suggest using $\hat{C}_{1}=-1$. Then for the eight unknowns we have in the semi-infinite case

$$
\begin{aligned}
C_{0} y_{0} & +C_{1}\left(\nu_{1}-\mu_{1}^{\prime} y_{0}\right)+C_{2}\left(\nu_{2}+\mu_{1}^{\prime 2} y_{0}\right)+C_{3}\left(\nu_{3}-\mu_{1}^{\prime 3} y_{0}\right) \\
& +C_{4}\left(\nu_{4}+\mu_{1}^{\prime 4} y_{0}\right)+\hat{C}_{0} \lambda_{0}+\hat{C}_{1} \lambda_{1}+\hat{C}_{2} \lambda_{2}+\hat{C}_{3} \lambda_{3}=0
\end{aligned}
$$

along with the seven equations (eight unknowns)

$$
\begin{aligned}
C_{0}\left(\nu_{s}\right. & \left.+\mu_{1}^{\prime} \nu_{s-1}\right)+C_{1}\left(\nu_{s+1}+\mu_{1}^{\prime} \nu_{s}\right)+C_{2}\left(\nu_{s+2}+\mu_{1}^{\prime} \nu_{s+1}\right)+ \\
& +C_{3}\left(\nu_{s+3}+\mu_{1}^{\prime} \nu_{s+2}\right)+C_{4}\left(\nu_{s+4}+\mu_{1}^{\prime} \nu_{s+3}\right)+\hat{C}_{0}\left(\lambda_{s}+\mu_{1}^{\prime} \lambda_{s-1}\right) \\
& +\hat{C}_{1}\left(\lambda_{s+1}+\mu_{1}^{\prime} \lambda_{s}\right)+\hat{C}_{2}\left(\lambda_{s+2}+\mu_{1}^{\prime} \lambda_{s+1}\right)+\hat{C}_{3}\left(\lambda_{s+3}+\mu_{1}^{\prime} \lambda_{s+2}\right)=0 . \\
& \left(s=1,2, \cdots, 7 ; \hat{C}_{1}=-1, \lambda_{s}=E(x+1)_{s}, \nu_{s}=E\left\{(x+1)^{s}-x^{s}\right\}\right)
\end{aligned}
$$




\subsection{Examples}

\subsubsection{Discrete Pseudo-Normal Distribution}

Take $\mu_{1}^{\prime}=0, \mu_{2}=1, \mu_{3}=0, \mu_{4}=3, \mu_{5}=0$ and $\mu_{6}=15$. The model is

$$
\begin{gathered}
y_{r}=\left(1+\frac{\left(\hat{C}_{0}\right)-x+\hat{C}_{2} x^{2}}{C_{0}+C_{1} x+C_{2} x^{2}}\right) y_{r-1} . \\
\left(r=0, \pm 1, \pm 2, \cdot \hat{C}_{0}=\alpha-\mu_{1}^{\prime}\right)
\end{gathered}
$$

Evaluated values are

$$
\left\{\begin{array}{l}
C_{0}=0.785715=11 / 14 \\
C_{1}=0.428572=6 / 14 \\
C_{2}=0.071429=1 / 14 \\
\hat{C}_{0}=0.5 \\
\hat{C}_{2}=0 .
\end{array}\right.
$$

This is identical to the earlier case 3.2 .2 with $\hat{C}_{2}$ taken to be zero.

\subsubsection{Discrete Pseudo-Normal with the First 8 Moments}

The equations in matrix form are:

$$
\begin{gathered}
{\left[\begin{array}{rrrrrr}
0 & 1 & 1 & 4 & 7 & 1 \\
1 & 1 & 4 & 7 & 26 & 1 \\
1 & 4 & 7 & 26 & 61 & 2 \\
4 & 7 & 26 & 61 & 232 & 4 \\
7 & 26 & 61 & 232 & 659 & 10 \\
26 & 61 & 232 & 659 & 2610 & 26
\end{array}\right]\left[\begin{array}{l}
C_{0} \\
C_{1} \\
C_{2} \\
C_{3} \\
C_{4} \\
\hat{C}_{0}
\end{array}\right]=\left[\begin{array}{r}
1 \\
2 \\
4 \\
10 \\
26 \\
76
\end{array}\right]} \\
k_{r}=\left(1+\frac{\hat{C}_{0}-r}{\sum_{s=0}^{4} C_{s} r^{s}}\right), \quad(r=0, \pm 1, \pm 2, \cdots)
\end{gathered}
$$

The solution is

$$
k_{r}=\phi(-R) / \phi(R), \quad(R=r-1 / 2)
$$

and

$$
\phi(R)=7430.0625+3710 R+605.5 R^{2}-7 R^{4},
$$

with a negative coefficient of $R^{4}$. The probabilities are

\begin{tabular}{|ccccccc|}
\hline$r$ & 0 & \pm 1 & \pm 2 & \pm 3 & \pm 4 & \pm 5 \\
\hline$y_{r}$ & 0.4006 & 0.2432 & 0.05419 & 0.004454 & 0.0001375 & $0.517 \times 10^{-6}$ \\
\hline
\end{tabular}

with sum $1.0046 ; y_{6}$ is negative.

A curiosity is the comparison of this case with the approximation $y_{r} \approx e^{-\frac{1}{2} r^{2}} / \sqrt{2 \pi}$, for which $y_{r} / y_{r-1}=e^{\frac{1}{2}-r}$. 


\begin{tabular}{|ccc|}
\hline \multicolumn{3}{|c|}{ Ratio $y_{r} / y_{r-1}$} \\
\hline$r$ & Discrete Model & Normal Approx. \\
\hline 1 & 0.606825 & 0.606531 \\
2 & 0.222874 & 0.223130 \\
3 & 0.082410 & 0.082085 \\
4 & 0.030319 & 0.030197 \\
5 & 0.003759 & 0.011109 \\
-0.5 & 2.718164 & $2.718282(\mathrm{e})$ \\
\hline
\end{tabular}

\subsubsection{Discrete Pseudo-Normal with the First 10 Moments}

We take $R=r-1 / 2$ and

$$
\begin{aligned}
p_{s} & =\sum_{-\infty}^{\infty}\left(y_{r}-y_{r-1}\right) R^{s}=0 \text { if } s \text { is even } \\
& =\sum_{-\infty}^{\infty} y_{r}\left[(r-1 / 2)^{s}-(r+1 / 2)^{s}\right] \\
q_{s} & =\sum_{-\infty}^{\infty} y_{r-1} R^{s}=\sum_{-\infty}^{\infty}(r+1 / 2)^{s} y_{r}
\end{aligned}
$$

Thus $p_{2 s+1}=-2 q_{2 s+1}$. New "enriched model" is

$$
y_{r}-y_{r-1}=y_{r-1}\left(\frac{-R-2 A_{3}^{*} R^{3}}{A_{0}^{*}+A_{1}^{*} R+A_{2}^{*} R^{2}+A_{3}^{*} R^{3}+A_{4}^{*} R^{4}}\right)
$$

and

$$
\begin{gathered}
k_{r}=\frac{A_{0}^{*}+\left(A_{1}^{*}-1\right) R+A_{2}^{*} R^{2}-A_{3}^{*} R^{3}+A_{4}^{*} R^{4}}{A_{0}^{*}+A_{1}^{*} R+A_{2}^{*} R^{2}+A_{3}^{*} R^{3}+A_{4}^{*} R^{4}}=\Phi(R) . \\
(R=r-1 / 2 ; r=0, \pm 1, \pm 2, \cdots)
\end{gathered}
$$

Then $\Phi(R) \Phi(-R)=1$ provided $A_{1}^{*}=1-A_{1}^{*}$ or $A_{1}^{*}=1 / 2$.

The equations in matrix form with four parameters are:

$$
\left[\begin{array}{rrrr}
p_{1} & p_{3} & 2 q_{4} & p_{5} \\
p_{3} & p_{5} & 2 q_{6} & p_{7} \\
p_{5} & p_{7} & 2 q_{8} & p_{9} \\
p_{7} & p_{9} & 2 q_{10} & p_{11}
\end{array}\right]\left[\begin{array}{c}
A_{0}^{*} \\
A_{2}^{*} \\
A_{3}^{*} \\
A_{4}^{*}
\end{array}\right]=\left[\begin{array}{l}
-q_{2} \\
-q_{4} \\
-q_{6} \\
-q_{8}
\end{array}\right] .
$$

The solution is

$$
\left\{\begin{array}{l}
A_{0}^{*}=31025 /(8 \cdot 3878) \\
A_{1}^{*}=1939 / 3878 \\
A_{2}^{*}=411 / 3878 \\
A_{3}^{*}=44 / 3878 \\
A_{4}^{*}=2 / 3878
\end{array}\right.
$$




\subsubsection{Discrete Pseudo-Normal with the First 16 Moments}

The "enriched model" is

$$
\begin{array}{r}
k_{r}=\frac{A_{0}-A_{1} R+A_{2} R^{2}-A_{3} R^{3}+A_{4} R^{4}-A_{5} R^{5}+A_{6} R^{6}}{A_{0}+A_{1} R+A_{2} R^{2}+A_{3} R^{3}+A_{4} R^{4}+A_{5} R^{5}+A_{6} R^{6}} . \\
(R=r-1 / 2 ; r=0, \pm 1, \pm 2, \cdots)
\end{array}
$$

The equations in matrix form with six parameters are:

$$
\left[\begin{array}{rrrrrr}
p_{1} & p_{3} & 2 q_{4} & p_{5} & 2 q_{6} & p_{7} \\
p_{3} & p_{5} & 2 q_{6} & p_{7} & 2 q_{8} & p_{9} \\
p_{5} & p_{7} & 2 q_{8} & p_{9} & 2 q_{10} & p_{11} \\
p_{7} & p_{9} & 2 q_{10} & p_{11} & 2 q_{12} & p_{13} \\
p_{9} & p_{11} & 2 q_{12} & p_{13} & 2 q_{14} & p_{15} \\
p_{11} & p_{15} & 2 q_{14} & p_{15} & 2 q_{16} & p_{17}
\end{array}\right]\left[\begin{array}{c}
A_{0} \\
A_{2} \\
A_{3} \\
A_{4} \\
A_{5} \\
A_{6}
\end{array}\right]=\left[\begin{array}{r}
-q_{2} \\
-q_{4} \\
-q_{6} \\
-q_{8} \\
-q_{10} \\
-q_{12}
\end{array}\right] .
$$

The solution is

$$
\left\{\begin{array}{l}
A_{0}=1.00000002049 \\
A_{1}=0.5 \\
A_{2}=0.113276449857 \\
A_{3}=0.014971578751 \\
A_{4}=0.001224410918 \\
A_{5}=0.000059007755 \\
A_{6}=0.000001311283 .
\end{array}\right.
$$

The probabilities are

\begin{tabular}{|clll|}
\hline$r$ & with 10 moments & with 16 moments & $e^{-r^{2} / 2} / \sqrt{2 \pi}$ \\
\hline 0 & 0.39894030 & 0.39894228 & 0.39894228 \\
\pm 1 & 0.24197226 & 0.24197072 & 0.24197072 \\
\pm 2 & 0.05399025 & 0.05399097 & 0.05399097 \\
\pm 3 & 0.00443204 & 0.00443185 & 0.00443185 \\
\pm 4 & 0.00013381 & 0.00013383 & 0.00013383 \\
\pm 5 & 0.000001486 & 0.000001487 & 0.000001487 \\
\hline$\sum$ & 1.0000 & 1.0000 & 1.0000 \\
\hline
\end{tabular}

Note that $y_{-1 / 2} / y_{-3 / 2}$ for the $4,10,16$ moments model has the values $2.697,2.718278$, and 2.718281840 respectively as approximants to $e$.

\subsubsection{Discrete Bessel Distribution with the First 10 Moments}

We consider the case with $\theta_{1}=\theta_{2}=1 / 2$. The first 10 moments are $0,1,0,4,0,31,0$, 379,0 , and 6556 . The basic model with four moments is

$$
y_{r}=\left[\frac{(2 R-4)^{2}+11}{(2 R+4)^{2}+11}\right] y_{r-1} \quad(R=r-1 / 2)
$$


and "enriched model with 10 moments is

$$
y_{r}=\left(\frac{A_{0}-A_{1} R+A_{2} R^{2}-A_{3} R^{3}+A_{4} R^{4}}{A_{0}+A_{1} R+A_{2} R^{2}+A_{3} R^{3}+A_{4} R^{4}}\right)^{y_{r-1}} .
$$

The solution is

$$
A_{0}=1145 /(8 \cdot 236), \quad A_{1}=118 / 236, \quad A_{2}=115 / 236, \quad A_{3}=24 / 236, \quad A_{4}=2 / 236 .
$$

\begin{tabular}{|c|c|c|c|c|}
\hline & Basic Model & 10 moments & 16 moments & $e^{-1} I_{r}(1)$ \\
\hline$r$ & $y_{r}$ & $y_{r}$ & $y_{r}$ & $y_{r}$ \\
\hline 0 & 0.422312 & 0.455065 & 0.464351 & 0.465760 \\
\hline \pm 1 & 0.234617 & 0.213919 & 0.208621 & 0.207910 \\
\hline \pm 2 & 0.046923 & 0.049170 & 0.049910 & 0.049939 \\
\hline \pm 3 & 0.006120 & 0.008246 & 0.008177 & 0.008155 \\
\hline \pm 4 & 0.000927 & 0.001026 & 0.001007 & 0.001007 \\
\hline \pm 5 & 0.000185 & 0.000098 & 0.000100 & 0.000100 \\
\hline \pm 6 & 0.000047 & 0.000008 & 0.000008 & 0.000008 \\
\hline \pm 7 & 0.000014 & 0.000001 & 0.000001 & 0.000001 \\
\hline \pm 8 & 0.000005 & & & \\
\hline \pm 9 & 0.000002 & & & \\
\hline \pm 10 & 0.000001 & & & \\
\hline$\sum y_{r}$ & 1.000000 & 1.000000 & 1.000000 & \\
\hline
\end{tabular}

\section{THE ALGEBRAIC STRUCTURE FOR THE DOUBLY BOUNDED CASE}

\subsection{First Order Equations}

From the fundamental moment parameter in (3) we deduce the following:

$$
\begin{aligned}
\sum_{r=1}^{N}\left(y_{r}-y_{r-1}\right)\left(x^{s}-T x^{s-1}\right) & =-l_{s} \quad(s=1,2, \ldots) \\
& =-\left[\hat{l}_{s}+(-1)^{s} \mu_{1}^{s-1}(N+1) y_{0}\right] \\
\sum_{r=1}^{N} y_{r-1}\left(x^{s}-T x^{s-1}\right) & =n_{s} \\
& =\lambda_{s}-T \lambda_{s-1} \\
\sum_{r=1}^{N}\left(y_{r}-y_{r-1}\right)\left(x^{s}+\mu_{1}^{\prime} x^{s-1}\right) & =-k_{s} \\
& =-\left[\hat{k}_{s}-T^{s-1}(N+1) y_{N}\right] \\
\sum_{r=1}^{N} y_{r-1}\left(x^{s}+\mu_{1}^{\prime} x^{s-1}\right) & =m_{s}
\end{aligned}
$$




$$
=\hat{m}_{s}-T^{s-1}(N+1) y_{N}
$$

where

$$
\left\{\begin{array}{l}
\hat{l}_{s}=\nu_{s}-T \nu_{s-1} \\
\hat{k}_{s}=\nu_{s}+\mu_{1}^{\prime} \nu_{s-1} \\
\hat{m}_{s}=\lambda_{s}+\mu_{1}^{\prime} \lambda_{s-1}
\end{array}\right.
$$

\subsection{Second Order Equations}

$$
\begin{aligned}
\sum_{r=1}^{N}\left(y_{r}-y_{r-1} 0\left(x+\mu_{1}^{\prime}\right)(x-T) x^{s-2}\right. & =\sum_{r=1}^{N}\left(y_{r}-y_{r-1}\right)\left[x^{2}+\left(\mu_{1}^{\prime}-T\right) x-\mu_{1}^{\prime} T\right] x^{s-2} \\
& =-\nu_{s}+\left(T-\mu_{1}^{\prime}\right) \nu_{s-1}+!_{1}^{\prime} T \nu_{s-2} \\
& =-L_{s} \\
\sum_{r=1}^{N} y_{r-1}\left(x+\mu_{1}^{\prime}\right)(x-T) x^{s-2} & =\sum_{r=1}^{N} y_{r-1} x^{s-2}\left[x^{2}+\left(\mu_{1}^{\prime}-T\right) x-\mu_{1}^{\prime} T\right] \\
& =M_{s} \quad(s=2,3, \cdots) \\
& =\lambda_{s}+\left(\mu_{1}^{\prime}-T\right) \lambda_{s-1}-\mu_{1}^{\prime} T \lambda_{s-2}
\end{aligned}
$$

It will be seen from (15-16) that the moment-parameter involved eliminates $y_{N}$ as an explicit component; similarly the moment operator in (17-18) eliminates $y_{0}$. Again the "quadratic" moment parameter in (19-20) eliminates $y_{0}$ and $y_{N}$ as far as they appear explicitly. These formulas show that, in the general discrete model, the parameters of the multiplier $k_{s}$ will be ratios of polynomials each involving a constant, and terms in $y_{0}, y_{N}$ and $y_{0} y_{N}$ only. We illustrate using the basic model for which

$$
k_{r}=1+\frac{\hat{C}_{0}-x}{C_{0}+C_{1} x+C_{2} x^{2}} . \quad(r=1,2, \cdots, N)
$$

Using (15-20) the equations now become

$$
\left\{\begin{array}{c}
{\left[\hat{l}_{1}-(N+1) y_{0}\right] C_{0}+\left[\hat{l}_{2}+\mu_{1}^{\prime}(N+1) y_{0}\right] C_{1}+\left[\hat{l}_{3}-\mu_{1}^{\prime 2}(N+1) y_{0}\right] C_{2}+n_{1} \hat{C}_{0}=n_{2}} \\
{\left[\hat{k}_{1}-(N+1) y_{N}\right] C_{0}+\left[\hat{k}_{2}-T(N+1) y_{N}\right] C_{1}+\left[\hat{k}_{3}-T(N+1) y_{N}\right] C_{2}} \\
\quad+\left[\hat{m}_{1}-(N+1) y_{N}\right] \hat{C}_{0}=\hat{m}_{2}-T(N+1) y_{N} \\
L_{2} C_{0}+L_{3} C_{1}+L_{4} C_{2}+M_{2} \hat{C}_{0}=M_{3} \\
L_{3} C_{0}+L_{4} C_{1}+L_{5} C_{2}+M_{3} \hat{C}_{0}=M_{4}
\end{array}\right.
$$

(Note that the powers of $\mu_{1}^{\prime}$ in the first equation of (22) alternate in sign, whereas those of $T$ in the second equation do not.)

Th last two equations do not contain $y_{0}$ or $y_{N} ; y_{0}$ is linear in the first equation, and $y_{N}$ linear in the second equation. Again it will be seen that the first three columns of the underlying matrix refer to the denominator of $k_{r}$. Clearly the determinant of the system $\Delta$ involves only a constant, $y_{0}, y_{N}$ and $y_{0} y_{N} ;$ similarly for the numerators of $C_{0}$, 
$C_{1}, C_{2}$ and $\hat{C}_{0}$. Algebraic solutions of (22) and similar generalizati ons should present no problem using a computer language such as Maple; one would sisume the existence of moments, and non-singular matrices. Numerically one would use $y_{0}^{*}$ and $y_{N}^{*}$ as seeds in (22) and attempt iterative solutions of $\sum_{1}^{N} y_{r}=1$, and $y_{N}=y_{N} \cdot y_{N-1} \cdots y_{0}$.

To study generalization of (21); suppose the denominator is $C_{0}+C_{1} x+\cdots C_{\lambda} x^{\lambda}$, and the numerator $\hat{C}_{0}+\hat{C}_{1} x+\cdots+\hat{C}_{u} x^{u}$. We elect which parameter shall be unity; suppose it is $\hat{C}_{u}$ for simplicity; then $\lambda+u+1$ equations are needed. The linearized model is then operated on by (15) and (16); then (17) and (18) and finally (19) and (20). The first $\lambda+1$ columns of the matrix will contain $(\hat{l}, \hat{k}, L)$, and the remaining $u$ columns will contain $(n, m, M)$.

\section{THE EXPONENTIAL AND THE NORMAL}

\subsection{The Continued Fraction}

Recalling that for the discrete normal we assume there is the approximation $y_{r}=$ $e^{-r^{2} / 2} / \sqrt{2 \pi}$, so that $k_{r}=y_{r} / y_{r-1}=e^{1 / 2-r}=e^{R}$. But there is a continued fraction (c.f.) for $k_{r}$, namely

$$
e^{R}=\frac{1}{1-} \frac{R}{1+} \frac{R}{2-} \frac{R}{3+} \frac{R}{2-} \frac{R}{5+} \cdots \quad(|R|<\infty)
$$

with convergents $\chi_{s}(R) / \omega_{s}(R)$, where

\begin{tabular}{lcc}
$s$ & $\chi_{s}(R)$ & $\omega_{s}(R)$ \\
\hline 0 & 0 & 1 \\
1 & 1 & 1 \\
2 & 1 & $1-R$ \\
3 & $2+R$ & $2-R$ \\
4 & $6+2 R$ & $12-4 R+R^{2}$ \\
5 & $12+6 R+R^{2}$ & $60-36 R+9 R^{2}-R^{3}$ \\
6 & $60+24 R+3 R^{2}$ & $1680-840 R+180 R^{2}-20 R^{3}+R^{4}$
\end{tabular}

It will be seen that there is a subset of convergents of the form $[\phi(R) / \phi(-R)], \phi(\cdot)$ being a polynornial. In fact the subset is $\chi_{4 s+1}(R) / \omega_{4 s+1}(R)$. And these consist of polynomials which are positive for all real $R$; they are thus compatible with the desired structure of $k_{r}$.

It is of some interest to note the definite integral form (not apparently given in the standard textbooks on c.f.s):

$$
\begin{aligned}
\chi_{2 s}(R) & =\frac{1}{(s--1) !} \int_{0}^{\infty} e^{-t} t^{s}(t+R)^{s-1} d t \\
\omega_{2 s}(R) & =\frac{1}{(s-1) !} \int_{0}^{\infty} e^{-t} t^{s-1}(t-R)^{s} d t \\
\chi_{2 s+1}(R) & =\frac{1}{s !} \int_{0}^{\infty} e^{-t} t^{s}(t+R)^{s} d t
\end{aligned}
$$




$$
\omega_{2 s+1}(R)=\frac{1}{s !} \int_{0}^{\infty} e^{-t} t^{s}(t-R)^{s} d t
$$

These can be proved by integration by parts and reference to the c.f. in (23).

\subsection{Relation to Whittaker Functions}

There are asymptotic forms for $R \rightarrow \infty$ and $R \rightarrow-\infty$. From Whittaker and Watson [11] we fi:st of ali consider positive $R$. Now

$W_{k, m}(z)=\frac{e^{-z / 2} z^{k}}{\Gamma(1 / 2-k+m)} \int_{0}^{10} e^{-t} t^{-k-1 / 2+m}(1+t / z)^{k-1 / 2+m} d t \quad(\Re(k-1 / 2+m) \leq 0)$

for all $z$ except negative reals. Hence

$$
\left\{\begin{array}{l}
\omega_{2 s}(-R)=R^{s-1 / 2} e^{R / 2} W_{1 / 2, s}(R) \\
\omega_{2 s+1}(-R)=R^{s} e^{R / 2} W_{0, s+1 / 2}(R) \quad(R>0)
\end{array}\right.
$$

When $R>0$ there are two components in the integrals. Here

$$
\begin{aligned}
\omega_{2 s}(R) & =\frac{1}{(s-1) !} \int_{0}^{\infty} e^{-t} t^{s-1}(t-R)^{s} d t \\
& =\frac{(-1)^{s}}{(s-1) !} \int_{0}^{R} e^{-t} t^{s-1}(R-t)^{s}+\frac{e^{-R}}{(s-1) !} \int_{0}^{\infty} e^{-t} t^{s}(R+t)^{s-1} d t
\end{aligned}
$$

The first term relates to the confluent hypergeometric

$$
M(a, b ; z)=\frac{\Gamma(b)}{\Gamma(b-a) \Gamma(a)} \int_{0}^{1} e^{z t} t^{a-1}(1-t)^{b-a-1} d t, \quad(\Re b>\Re a>0)
$$

and the second to Whittaker's $W$. In fact,

$$
\omega_{2 s}(R)=(-1)^{s} R^{2 s} \frac{\Gamma(s+1)}{\Gamma(2 s+1)} M(s, 2 s+1 ;-R)+s R^{s-1 / 2} e^{-R / 2} W_{-1 / 2, s}(R) . \quad(R<0)
$$

Similarly,

$$
\omega_{2 s+1}(R)=(-1)^{s} R^{2 s+1} \frac{\Gamma(s+1)}{\Gamma(2 s+2)} M(s+1,2 s+2 ;-R)+e^{-R / 2} R^{s} W_{0, s+1 / 2}(R)
$$

Whittaker and Watson [11] give the asymptotic form for $|R|$ large, the basic asymptotic being

$$
W_{k, m}(z) \sim e^{-z / 2} z^{\kappa} . \quad(|\arg z| \leq \pi-\alpha<\pi)
$$

In our present context it will be seen that

$$
\lim _{s \rightarrow \infty}\left(\frac{\chi_{4 s+1}}{\omega_{4 s+1}(-R)}\right)=e^{-R}=\lim _{s \rightarrow \infty}\left(\frac{\omega_{4 s+1}(R)}{\omega_{4 s+1}(-R)}\right)
$$




\subsection{Application to the Normal}

We can now set up the discrete normal not using moments but appropriate convergents of (23). For example, we may use

$$
k_{r}=k_{R}^{(5)}=\frac{12-6 R+R^{2}}{12+6 R+R^{2}}
$$

and the equation

$$
y_{0}\left[1+2 k_{1}^{(5)}+2 k_{2}^{(5)} k_{1}^{(5)}+\cdots\right]=1,
$$

to determine $y_{0}$. Note however there is a small error in using $\sum_{-\infty}^{\infty} y_{r}=1$; incidentally Aitken [1] gave $\sum_{-\infty}^{\infty} e^{-r^{2} / 2}=2.506628288$ (nearly) or $\sum_{-\infty}^{\infty} y_{r} \sim 1.000,000,005$.

An alternative is to assume $y_{0}=1 / \sqrt{2 \pi}$ and determine the sum from (24). For the former choice, $y_{0}=0.397466120$ and $1 / \sqrt{2 \pi} \sim 0.398942280$.

Higher order convergents, such as

$$
k_{r}^{4 s+1}=\omega_{4 s+1}(R) / \omega_{4 s+1}(-R) \quad(s=2.3 . \cdots)
$$

lead to using the limiting value $\exp (-R)$ so that $y_{r} / y_{0}$ is seen as the product

$$
\prod_{s=1}^{r} \exp [-(2 s-1) / 2]
$$

a very interesting interpretation, providing a link between c.f.s and the discrete normal.

\subsection{Moments of the Discrete Normal Distribution}

We use Hermite polynomials $\left\{H_{s}(x)\right\}$, an orthogonal system with respect to the basic normal density $\exp \left(-x^{2} / 2\right) / \sqrt{2 \pi}$, and for which

$$
\left\{\begin{array}{l}
H_{s}(x)=e^{-D_{x}^{2} / 2} x^{s}, \\
x^{s}=e^{D_{x}^{2} / 2} H_{s}(x) .
\end{array} \quad\left(D_{x} \equiv d / d x ; D_{x} H_{s}(x)=s H_{s-1}(x)\right)\right.
$$

Consider then the evaluation of

$$
h_{2 s}^{*}=\sum_{r=-\infty}^{\infty} \frac{H_{s}(r) e^{-r^{2} / 2}}{\sqrt{2 \pi}} . \quad(s=0,1, \ldots)
$$

Then the Euler summation formula (see Konrad Knopp), [4] shows that

$$
h_{2 s}^{*}=\int_{-\infty}^{\infty} \frac{H_{2 s}(x) e^{-x^{2} / 2} d x}{\sqrt{2 \pi}}+\Re_{1},
$$

where

$$
\Re_{1}=\int_{0}^{\infty} P_{3}(x) \frac{d^{3}}{d x^{3}}\left[\frac{H_{2 s}(x) e^{-x^{2} / 2}}{\sqrt{2 \pi}}\right] d x
$$


and

$$
P_{3}(x)=\sum_{n=1}^{\infty} \frac{2 \sin (2 n \pi x)}{(2 n \pi)^{3}} .
$$

Integration by parts, with assumptions regarding uniform convergence, leads to

$$
k_{2 s}^{*}=\delta_{s, 0}+h_{2 s}
$$

where

$$
h_{2 s}=2(-1)^{s} \sum_{t=1}^{\infty}(2 \pi t)^{2 s} e^{-2(\pi t)^{2}}
$$

For examples,

$$
\begin{gathered}
h_{0}^{*}=1+2\left(e^{-2 \pi^{2}}+e^{-8 \pi^{2}}+e^{-18 \pi^{2}}+\cdots\right), \\
h_{2}^{*}=-8 \pi^{2}\left(e^{-2 \pi^{2}}+2^{2} e^{-8 \pi^{2}}+3^{2} e^{-18 \pi^{2}}+\cdots\right),
\end{gathered}
$$

with regard to $h_{0}^{*}$. Dr. Robert Byers using a variable precision package "Derive", gives

$$
\sqrt{2 \pi} h_{0}^{*}=2.506,628,288,042,905,544,830,67,
$$

and

$$
\sqrt{2 \pi}\left(h_{0}^{*}-1\right)=0.000,000,013,411,905,042,414,91 .
$$

From (25) and (26) we now have the general formula for the central moments of the discrete normal distribution, namely

$$
\mu_{2 s}=\sum_{m=0}^{s} \frac{(2 s)^{(2 m)}}{2^{m} m !}\left(\frac{\delta_{2 s-2 m, 0}+h_{2 s-2 m}}{1+h_{0}}\right) .
$$

For examples,

$$
\begin{array}{r}
\mu_{2}=\left[h_{2}+\left(1+h_{0}\right)\right] /\left(1+h_{0}\right), \\
\mu_{4}=\left[h_{4}+6 h_{2}+3\left(1+h_{0}\right)\right] /\left(1+h_{0}\right) .
\end{array}
$$

\section{CONCLUDING REMARKS}

We have studied various aspects of the Pearson discrete model in generalized forms, including the doubly infinite case, semi-infinite case and the bounded case. The examples chosen have been directed at gaining insight into the models and their implementation. New interesting properties have been disccivered with respect to the normal case; on the one hand using the standard normal moments as against the corrected moments.

Applications to empirical data and estimation problems are not undertaken at this time.

\section{References}

[1] A.C. Aitken. Statistical Mathematics. Oliver and Boyd, Edinburgh, G.B., 1939. 
[2] G.H. Hansmann. On certain non-normal symmetrical frequency distributions. Biometrika, 26:1 :9-195, 1934.

[3] N.L. Johnson and S. Kotz. Discrete Distributions. Houghton Mifflin Company, Boston, 1969.

[4] Konrad Knopp. Theory and Application of Infinite Series. Blackie and Son Ltd., London and Glasgow, 1928.

[5] J.K. Ord. On a system of discrete distributions. Biometrika, 54:649-656, 1967.

[6] J.K. Ord. The discrete student's $t$ distribution. Annals of Math. Stat., 39:1513$1516,1968$.

[7] J.K. Ord. Families of Frequency Distributions. Charles Griffin, London, 1968.

[8] J.K. Ord. Pearson system of distributions. Encyclopedia of Statistical Sciences, 6 , 1985.

[9] K. Pearson. Early Statistical Papers. Cambridge University Press, Cambridge, England, 1948.

[10] J.G. Skellam. The frequency distribution of the difference of two poisson variates belonging to different populations. J.R.S.S., 109:296, 1946.

[11] E.T. Whittaker and G.N. Watson. Modern Analysis, 2nd edition. Cambridge University Press, Cambridge, England, 1915. 
ORNL/TM-11899

\section{INTERNAL DISTRIBUTION}

1. B. R. Appleton

2. C. K. Bayne

3. J. J. Beauchamp

4-8. K. O. Bowman

9. C. C. Brandt

10-11. R. T. Darland

12. D. M. Flanagan

13. E. L. Frome

14. T. L. Hebble

15. E. Leach

16. T. J. Mitchell

17. M. D. Morris

18. G. Ostrouchov
19-23. S. A. Raby

24. R. L. Schmoyer

25-29. R. F. Sincovec

30-34. R. C. Ward

35. D. A. Wolf

36. T. Wright

37. Central Research Library

38. ORNL Patent Office

39. K-25 Plant Library

40. Y-12 Technical Library /

Document Reference Station

41. Lahoratory Records - RC

42-43. Laboratory Records Department

\section{EXTERNAL DISTRIBUTION}

44. Shunichi Amari, Faculty of Engineering, University of Tokyo, Bunkyo-ku, Tokyo 113, Japan

45. George A. Barnard, Mill House, 54 Hurst Green, Brightlingsea, Colchester, Essex C07 0EH, United Kingdom

46. Roger W. Brockett, Division of A pplied sciences, Harvard University, Cambridge, MA 02138

47. Robert H. Byers Jr, Statistics and Data Branch, Centers for Disease Control, HIV/AIDS Division, 1509 Sheridan Walk NE, Atlanta, GA 30324

48. John Cavallini, Acting Director, Scientific Computing staff, Applied Mathematical Sciences, Office of Energy Research, U.S. Department of Energy, Washington, DC 20585

49. Gary A. Churchill, Plant Bleeding and Biometry Department, Cornell University, 337 Warren Hall, Ithaca, NY 14853

50. P. C. Consul, Mathematics and Statistics Department, University of Calgary, Calgary, T2N 1N4, Canada

51. Edwin L. Crow, U.S. Department of Commerce, Boulder Laboratories ITS.N3, 325 Broadway, Boulder, CO 80303-3328

52. John J. Dorning, Department of Nuclear Engineering Physics, Thornton Hall, McCormick Road, University of Virginia, Charlottesville, VA 22901

53. James B. Douglas, Statistics Department, University of New South Wales, Box 1, Kensington, NSW 2033, Australia

54. Max Eisenburg, Center for Indoor Air Research, 1099 Winterson Road, Suite 280, Linthicum, MD 21090 
55. Jerome Friedman, Department of Statistics, Sequoia Hall, Stanford University, Stanford, CA 94305

56. John Gurland, Statistics Department, University of Wisconsin, 12210 Dayton St, Madison, WI 53706

57. Kermit Hutcheson, Statistics Department, University of Georgia, Athens, GA 30602

58. N. L. Johnson, Statistics Department, University of North Carolina, Chapel Hill, NC 27599-3260

59-63. M. A. Kastenbaum, P.O.Box 315, Basye, VA 22810

64. A. D. Kemip, Statistics Department, University of St Andrews North Haugh, St Andrew KY16 9SS, Scotland

65. Samuel Kotz, Management Sciences and Statistics, University of Maryland, College Park, MD 20742

66. James E. Leiss, Rt. 2, Box 142C, Broadway, VA 22815

67. Neville Moray, Department of Mechanical and Industrial Engineering, University of Illinois, 1206 West Green Street, Urbana, IL 61801

68. T. Okuno, 17-8 Minami-cho 4, Tanashi, Tokyo, 188A, Japan

69. J. K. Ord, Management Science Department, Pennsylvania State University, 303 Bean Business Administration Bldg., University Park, PA 16802

70. Jerome Sacks, Department of Statistics, University of Illiinois, 101 Illini Hall, 725 Write Street, Champain, IL 61820

71-75. L. R. Shenton, University of Georgia, Athens, GA 30602

76. Daniel L. Solomon, Department of Statistics, North Carolina State University, P.O.Box 5457, Raleigh, NC 27650

77. Alan Stuart, 82 Somerset Road, Wimbledon, London SW19 5JX, United Kingdom

78. Werner Stuetzle, Department of Statistics, GN-22, University of Washington, Seattle, WA 98195

79. Ray A. Waller, S-1, Statistics, Los Alamos National Laboratory, P.O.Box 1663, Los Alamos, NM 87545

80. Mary F. Wheeler, Rice University, Depart of Mathematical Sciences, P.O.Box 1892, Houston, TX 77001

81. Alice Zeiger, Center for Indoor Air Research 1099 Winterson Road, Suite 280, Linthicum, MD 21090

82. Office of Assistant Manager for Energy Research and Development, U.S. Department of Energy, Oak Ridge Operations Office, P.O.Box 2001, Oak Ridge, TN $37831-8600$

83-92. Office of Scientific and Technical Information, P.O.Box 62, Oak Ridge, TN 37830 

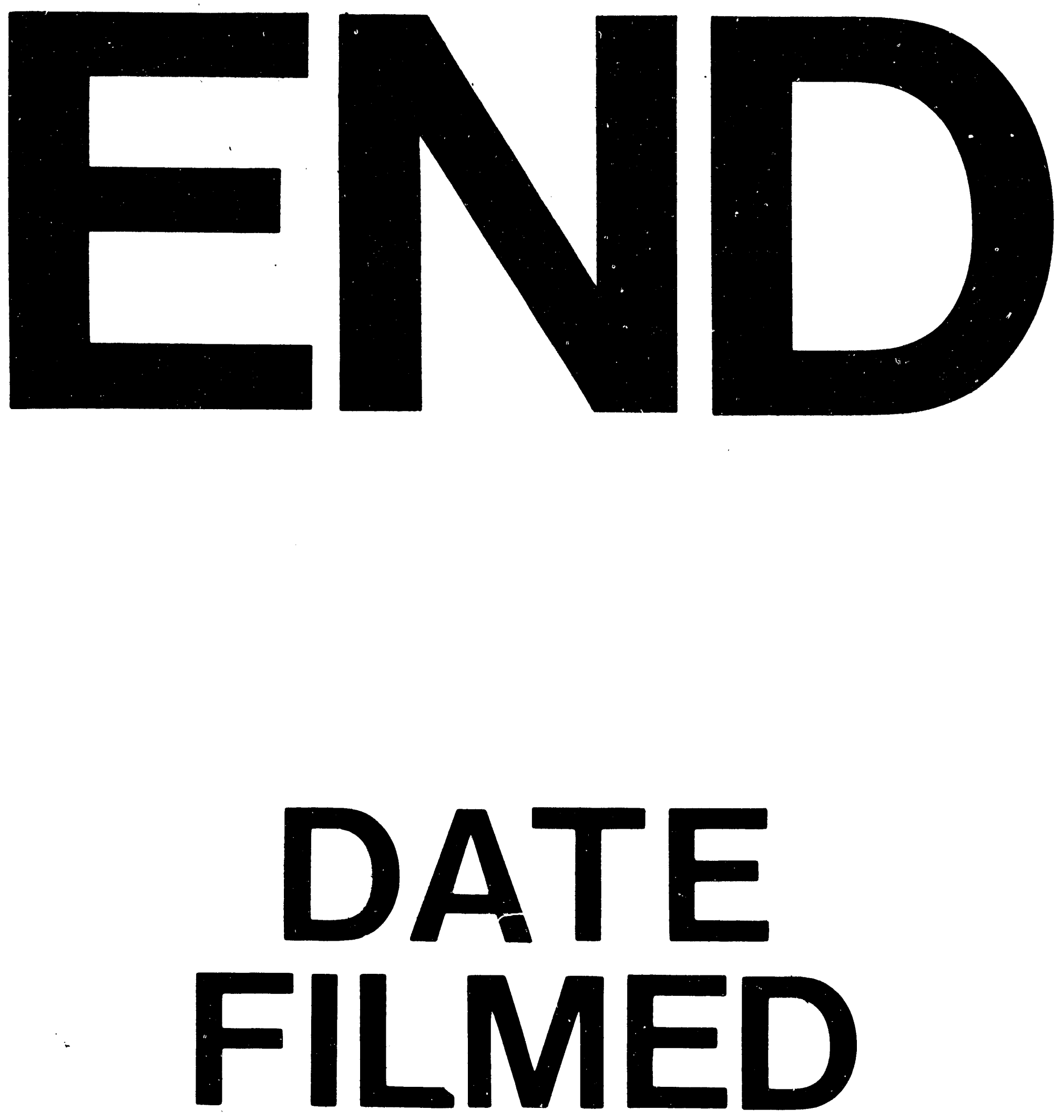

1

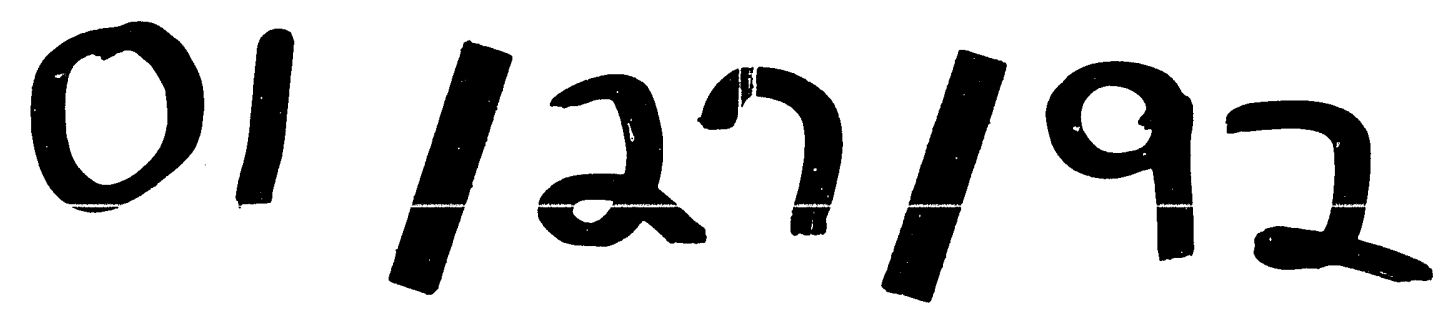


